\title{
A CLINICO-EPIDEMIOLOGICAL STUDY OF PSORIASIS IN A TERTIARY CARE HOSPITAL IN PATNA, BIHAR
}

\section{Dermatology}

Dr. Vikas Shankar

Dr. Alok Kumar*

Dr. Manadavi

M.B.B.S., M.D. (Skin \& VD), Assistant Professor, Department of Dermatology, Venereology and Leprosy, Patna Medical College and Hospital, Patna, Bihar.

M.B.B.S., M.D. (Skin \& VD), Senior Resident, Department of Dermatology, Venereology and Leprosy, Patna Medical College and Hospital, Patna, Bihar. *Corresponding Author

M.B.B.S., M.D. (Pharmacology, Final year), Junior Resident, Department of Pharmacology, Patna Medical College and Hospital, Patna, Bihar.

Dr. Ayushi M.B.B.S.

\section{Dr. Debarshi Jana}

Young Scientist (DST) Institute of Post-Graduate Medical Education and Research, A.J.C. Bose Road, Kolkata-700020, West Bengal, India.

\section{ABSTRACT}

Background: Psoriasis is a chronic inflammatory disease most commonly manifested by skin lesions on the elbows, knees, scalp, genitals, and trunk. The co-morbidities more commonly associated to psoriasis include psoriatic arthritis (PsA) and Crohn's disease, others being hyperlipidemia, diabetes mellitus, arterial hypertension, rheumatoid arthritis, obesity, ischemic heart disease, ulcerative colitis, reduced quality of life, depression and malignancy. Present study aims to study the prevalence of co- morbidity factors associated with psoriasis. Materials and methods: A hospital based cross sectional study was conducted in Dermatology outpatient department at a tertiary hospital in Patna, Bihar. Written informed consent was taken from the patients. Detailed clinical evaluation and relevant investigations were done for all patients. Data was analysed using SPSS software ver. 17. Results: A total of 86 psoriasis patients were included in the study. Male pre-dominance (65\%) was observed with majority of patients were between 30-50 years of age. Psoriasis was associated with a significantly increased prevalence for hypertension (46.51\%), hyperlipidemia (48.83\%), diabetes $(38.31 \%)$, cardiovascular disease $(10.46 \%)$, COPD $(9.30 \%)$, psoriatic arthritis $(29.06 \%)$, metabolic syndrome $(34.88 \%)$, Crohn's disease $(1.16 \%)$ and depression $(47.67 \%)$. Conclusion: The study revealed a male preponderance with peak incidence in third and fourth decade of life. We also observed a high prevalence of associated co-morbidites in patients of psoriasis which play a major role in diasease progression and hence should be addressed accordingly.

\section{KEYWORDS}

Cardiovascular Disease, Co-morbidities, Psoriatic Arthritis, Psoriasis.

\section{INTRODUCTION}

Psoriasis is a chronic inflammatory disease most commonly manifested by skin lesions on the elbows, knees, scalp, genitals, and trunk that has been estimated to affect $1 \%$ to $3 \%$ of the population worldwide. Psoriasis is considered as an immune-mediated inflammatory disorder (IMID), alongside other entities such as rheumatoid arthritis, Crohn's disease and multiple sclerosis.

Despite their distinct clinical presentation, these diseases share common features such as chronic course, inflammatory nature and several pathogenetic mechanisms, including a so-called Th1-like cytokine milieu in the affected tissue, dominated by interferon alpha and gamma along with TNF-alpha, interleukins (IL)- 2, 22 and 17.

Another shared feature of IMIDs is the association with other diseases, in particular cardiovascular diseases. The co-morbidities more commonly associated to psoriasis include psoriatic arthritis (PsA) and Crohn's disease, others being dyslipidaemia, diabetes mellitus, arterial hypertension, rheumatoid arthritis, obesity, ischemic heart disease, ulcerative colitis, reduced quality of life, depression and malignancy.

Psoriatic arthritis affects up to one-third of patients with psoriasis. It is a seronegative spondyloarthritis involving both peripheral joints and axial skeleton, and usually begins as enthesitis i.e. the inflammation at tendon insertion. Enthesopathy has also been reported to be very common in psoriasis patients without clinical signs or symptoms of arthritis. PsA runs a chronic fluctuating course and about $20 \%$ of patients develop a very destructive and disabling form. Psoriasis precedes the development of PsA in the vast majority of patients, without co-relation between skin disease severity and risk of developing PsA.

\section{MATERIALAND METHODS}

This hospital based cross-sectional study was conducted in Dermatology outpatient department at a tertiary hospital in Patna, Bihar for duration of 1 year. A total of 86 consecutive patients were included in the study who were clinically diagnosed as psoriasis.

Socio-demographic and detailed clinical history for each patient was collected in a pre-designed proforma. Patients were subjected to routine blood investigations, skin biopsy, chest $\mathrm{X}$-ray and bone $\mathrm{X}$ - ray. The other investigations were done depending upon patient's condition. Data was analysed using SPSS ver. 17.

\section{RESULTS}

A total of 86 psoriasis patients were identified in the study of which majority were in the age group $45-55$ years $(36 \%)$ which consisted of mainly males $(56 \%)$ (Table - 1). The commonest comorbidity found in our study was hyperlipidemia $(48.83 \%)$, an increased prevalence of hypertension $(46.51 \%)$ was also seen. Other co-morbid conditions observed were diabetes $(38.31 \%)$, metabolic syndrome $(34.88 \%)$, psoriatic arthritis $(29.06 \%)$, cardiovascular disease (10.46\%), COPD $(9.30 \%)$ and crohns disease $(1.16 \%)$. Depression $(47.67 \%)$ was reported in a great majority of psoriasis patients. Psoriasis was not associated with an increased risk of stroke or lymphoma (Table - 2).

Table-1: Distribution of Age and Gender.

\begin{tabular}{|c|c|c|c|c|c|c|}
\hline \multirow{2}{*}{$\begin{array}{l}\text { Age } \\
\text { (years) }\end{array}$} & \multicolumn{2}{|c|}{ Male } & \multicolumn{2}{|c|}{ Female } & \multicolumn{2}{|c|}{ Total } \\
\hline & $\mathbf{N}$ & $\%$ & $\mathbf{N}$ & $\%$ & $\mathbf{N}$ & $\%$ \\
\hline $18-25$ & 2 & $66.7 \%$ & 1 & $33.3 \%$ & 3 & $100.0 \%$ \\
\hline $25-35$ & 6 & $60.0 \%$ & 4 & $40.0 \%$ & 10 & $100.0 \%$ \\
\hline $35-45$ & 19 & $70.4 \%$ & 8 & $29.6 \%$ & 27 & $100.0 \%$ \\
\hline $45-55$ & 22 & $61.1 \%$ & 14 & $38.9 \%$ & 36 & $100.0 \%$ \\
\hline$>55$ & \begin{tabular}{|l}
7 \\
\end{tabular} & $70.0 \%$ & 3 & $30.0 \%$ & \begin{tabular}{|l|}
10 \\
\end{tabular} & \begin{tabular}{|l|}
$100.0 \%$ \\
\end{tabular} \\
\hline Total & 56 & $65.1 \%$ & 30 & $34.9 \%$ & 86 & $100.0 \%$ \\
\hline
\end{tabular}

Table-2: Distribution of various co- morbidities.

\begin{tabular}{|l|l|l|}
\hline Co-morbidities (n-86) & $\mathbf{N}$ & \% \\
\hline Hypertension & 40 & $46.5 \%$ \\
\hline Diabetes mellitus & 33 & $38.4 \%$ \\
\hline Cholesterol & 42 & $48.8 \%$ \\
\hline Cardiovascular diseases & 9 & $10.5 \%$ \\
\hline COPD & 8 & $9.3 \%$ \\
\hline Crohn's disease & 1 & $1.2 \%$ \\
\hline Psoriatic arthritis & 25 & $29.1 \%$ \\
\hline Metabolic syndrome & 30 & $34.9 \%$ \\
\hline Depression & 41 & $47.7 \%$ \\
\hline
\end{tabular}




\section{DISCUSSION}

Psoriasis is associated with a significantly increased risk of comorbidities, especially for those patients with moderate to severe disease. These health associations should be taken into consideration when evaluating the burdens of psoriasis and designing effective treatment plans. In the light of the co-morbidities associated with psoriasis, managing these patients should not be limited to their skin symptoms, but should also include a holistic approach. As in case of PsA, dermatologists are in the position to detect this developing comorbidity early. All relevant measures can be taken in a simple way in outpatient setting. The comprehensive investigation profile of psoriasis patients should include measurement of pulse and blood pressure, determination of BMI and measurement of fasting blood lipids and blood glucose.

Kaye, et al. found that psoriasis itself conferred a risk of developing diabetes as the cumulative incidence of diabetes in the psoriasis cohort was higher than comparison data (hazard ratio 1.33; confidence interval (CI) 1.25-1.42). The association between psoriasis and type 2 diabetes is even stronger. Numerous cross-sectional studies have shown that psoriasis, especially severe disease, confers a higher risk (up to 2.48) of diabetes. The increased prevalence of diabetes in patients with psoriasis appears to be independent of traditional diabetes risk factors such as obesity and dyslipidemia. The shared genetic background may also contribute to the susceptibility to both psoriasis and diabetes.

In a cross sectional study, Gisondi, et al. found that psoriasis patients had a higher prevalence of metabolic syndrome versus general dermatology patients after controlling for sex and age $(30.1 \%$ vs. $20.6 \%$; OR $1.65 ; 95 \%$ CI $1.16-2.35$ ) which is similar to our study. However, the most notable observations made by Gefland JM, et al. are two large retrospective cohort studies using the General Practice Research Database that involved almost 130,000 patients with psoriasis. In these recent studies, the risk of myocardial infarction was $1.29(95 \%$ CI 1.14-1.46) for 30-year-old patients with mild psoriasis and 3.10 (95\% CI 1.98-4.86) for 30- year-old patients with severe psoriasis and the hazard ratio for stroke was 1.06 (95\% CI 1.0$1.1)$ and 1.43 (95\% CI 1.1-1.9) for mild and severe psoriasis, respectively.

Moreover, the dermatologist should be aware of the importance of recommending the patient lifestyle modifications including a low calorie diet, which may supplement the pharmacological treatment of obese patients. Weight loss, through calorie restriction, could improve metabolic comorbidities including decreasing insulin resistance, reducing serum lipids and reducing blood pressure levels. Furthermore, losing weight may improve psoriasis. Several case studies have shown that weight loss from gastric bypass surgery results in remission of psoriasis. Likewise, in a recent controlled study we showed that moderate weight loss (i.e. $5-10 \%$ of body weight) increases the therapeutic response to a low dose of cyclosporine in obese patients with moderate to severe chronic plaque psoriasis. This suggested that lifestyle modifications may supplement the pharmacological treatment administered to obese psoriasis patients. Weight loss, through calorie restriction, induces decreases in insulin, leptin, CRP and MCP-1, and increases adiponectin levels, resulting in an anti- inflammatory effect.

\section{CONCLUSION}

The study revealed a male preponderance with peak incidence in third and fourth decade of life. We also observed a high prevalence of associated co-morbidites in patients of psoriasis which play a major role in diasease progression and hence should be addressed accordingly.

\section{REFERENCES}

1. Cohen AD, Dreiher J, Shapiro Y, Vidavsky L, Vardy DA, Davidovici B, et al. Psoriasis and diabetes: a population- based cross-sectional study. J Eur Acad Dermatol Venereol., 2008; $22: 585-9$.

2. Gelfand JM, Dommasch ED, Shin DB, Azfar RS, Kurd SK, Wang X, et al. The risk of stroke in patients with psoriasis. J Invest Dermatol., 2009; 129: 2411-8.

3. Gelfand JM, Neimann AL, Shin DB, Wang X, Margolis DJ, Troxel AB. Risk of myocardial infarction in patients with psoriasis. JAMA, 2006; 296: 1735-41.

4. Gisondi P, Del Giglio M, Di Francesco V, Zamboni M, Girolomoni G. Weight loss makes obese patients with moderate to severe chronic plaque psoriasis highly responsive to low dose cyclosporine: a randomized, controlled, investigator blinded, clinical trial. Am J Clin Nutr., 2008; $88: 1242-7$.

5. Gisondi P, Girolomoni G, Sampogna F, Tabolli S, Abeni D. Prevalence of psoriatic arthritis and joint complaints in a large population of Italian patients hospitalised for arthritis and joint complaints in a large pop

6. Gisondi P, Tessari G, Conti A, Piaserico S,Schianchi S, Peserico A, et al. Prevalence of metabolic syndrome in patients with psoriasis:a hospital-based case-control study. $\mathrm{Br} J$ Dermatol.,2007; 157:68-73.

7. Gisondi P, Tinazzi I, El-Dalati G, Gallo M, Biasi D, Barbara LM, et al Lower limb enthesopathy in patients with psoriasis without clinical signs of arthropathy: a hospitalbased case- control study. Ann Rheum Dis., 2008; 67: 26-30.

8. Gudjonsson JE, Elder JT. Psoriasis: epidemiology. Clin Dermatol., 2007; 25: 535-46.

9. Higa-Sansone G, Szomstein S, Soto F, Brasecsco O, Cohen C, Rosenthal RJ. Psoriasis remission after laparoscopic Roux-en-Y gastric bypass for morbid obesity. Obes Surg., 2004; 14: 1132-4.

10. Husted JA, Tom BD, Farewell VT, Schentag CT, Gladman DD. A longitudinal study of the effect of disease activity and clinical damage on physical function over the course of psoriatic arthritis: does the effect change over time? Arthritis Rheum. 2007:56:840-9.

1. Kaye JA, Li L, Jick SS. Incidence of risk factors for myocardial infarction and other Kaye JA, Li L, Jick SS. Incidence of risk factors for myocardial infarction and
vascular diseases in patients with psoriasis. Br J Dermatol., 2008; 159: 895-902.

12. Lowes MA, Bowcock AM, Krueger JG. Pathogenesis and therapy of psoriasis. Nature, 2007; 445: 866-73.

13. Neimann AL, Shin DB, Wang X, Margolis DJ, Troxel AB, Gelfand JM. Prevalence of cardiovascular risk factors in patients with psoriasis J Am Acad Dermatol., 2006; 55 829-35.

14. Paolo Gisondi, Anna Ferrazzi, Giampiero Girolomoni. Metabolic Comorbidities and Psoriasis. Acta Dermatovenerol Croat., 2010; 18(4): 297-304.

15. Wilson FC, Icen M, Crowson CS, McEvoy MT, Gabriel SE, Kremers HM. Incidence an clinical predictors of psoriatic arthritis in patients with psoriasis: a population-based clinical predictors of psoriatic arthritis in p
study. Arthritis Rheum., 2009; 61:233-9.

16. Wolf N, Quaranta M, Prescott NJ, Allen M, Smith R, Burden AD, et al. Psoriasis is associated with pleiotropic susceptibility loci identified in type II diabetes and Crohn disease. J Med Genet., 2008; 45: 114-6. 